\title{
Preparation of mesoporous MgO-templated carbons from phenolic resin and their applications for electric double-layer capacitors
}

\author{
LIU WenJie, WANG ChengYang*, WANG JiuZhou, JIA Fan, ZHENG JiaMing \& CHEN MingMing \\ Key Laboratory for Green Chemical Technology of Ministry of Education, School of Chemical Engineering and Technology, Tianjin University, \\ Tianjin 300072, China
}

Received May 3, 2012; accepted June 23, 2012

\begin{abstract}
Mesoporous carbons were synthesized using thermoplastic phenolic resin (PF) as carbonaceous precursor and magnesium citrate as template precursor. Pore structure was determined as ink-bottle-like geometry through TEM, $\mathrm{N}_{2}$ adsorption analysis combined with TG curves. The porous carbons prepared were then applied as electrode material for electric double-layer capacitors. The capacitor performance was examined in $30 \mathrm{wt} \% \mathrm{KOH}$ aqueous solution by cyclic voltammetry and galvanostatic charge/discharge measurements. The carbon prepared with $\mathrm{MgO} / \mathrm{PF}$ mass ratio of $8 / 2$ had a BET surface area of $1920 \mathrm{~m}^{2} \mathrm{~g}^{-1}$ and exhibited a capacitance of $220 \mathrm{~F} \mathrm{~g}^{-1}$ at a current density of $50 \mathrm{~mA} \mathrm{~g}^{-1}$. Besides, the carbon with the ratio of $4 / 6$ had the optimize proportion of mesopores, which ensures its good rate performance that up to $98.3 \%$, expressed as the ratio of the capacitance measured at 1000 $\mathrm{mA} \mathrm{g}^{-1}$ against that at $50 \mathrm{~mA} \mathrm{~g}^{-1}$.
\end{abstract}

mesoporous, EDLC, MgO-templated carbon

Citation: Liu W J, Wang C Y, Wang J Z, et al. Preparation of mesoporous MgO-templated carbons from phenolic resin and their applications for electric double-layer capacitors. Chin Sci Bull, 2013, 58: 992-997, doi: 10.1007/s11434-013-5670-0

Porous carbons have attracted great attention because of their hydrophobic surface nature, high surface area, large pore volumes, chemical inertness, as well as good thermal and mechanical stability [1]. For the excellent physical and chemical property, porous carbons have been used in various fields, the nature of which depends on the pore size of carbon materials. For application as electrode of electric double-layer capacitor (EDLC), carbons should have mesopores for transferring electrolyte ions and micropores for adsorbing electrolyte ions to form electric double layer [2]. Thus, the preparation of carbons containing both mesopores and micropores has sparked enormous interest.

Ordered mesoporous carbons with different pore sizes and morphologies have been prepared by casting carbon frameworks with mesoporous silica templates [3-5]. However, template removal for this preparation method requires the employment of corrosive acids, making large-scale production difficult. A promising preparation method was

*Corresponding author (email: cywang@tju.edu.cn) recently reported that involves coating ceramic substrates with thermoplastic precursors [6-8]. Among various substrates, $\mathrm{MgO}$ and organic magnesium salt, such as magnesium citrate, magnesium acetate, magnesium gluconate, were proved to be ideal because the pyrolysis product, $\mathrm{MgO}$, exhibit perfect performance: chemical and thermal stability, no structural and compositional changes, no reaction with carbon up to carbonization temperature of carbon precursors, and easily dissolving into a diluted acidic solution $[9,10]$. What's more, this preparation method was proved to be energy-saving because nanoporous carbons were prepared without any stabilization and activation processes starting from thermoplastic precursors in this method [11-15].

Although many carbon precursors have been selected to investigate various kinds of $\mathrm{MgO}$-templated carbons, the interaction between carbon precursors and magnesium precursors during carbonization has not been properly discussed and the pore morphology of resultant carbons has not formed final conclusion. In the present work, we selected thermoplastic phenolic resin as carbon precursor, for 
its purity, low cost and high carbon yield, and magnesium citrate as $\mathrm{MgO}$ template because the abundant carbonaceous formed during carbonization can prevent the cohesion of $\mathrm{MgO}$ particles effectively.

\section{Experimental}

\subsection{Carbon preparation}

Magnesium citrate $(\mathrm{MgCi}$, reagent grade, Tianjin Guangfu Fine Chemical Research Institute, China) with phenol formaldehyde resin (PF, novolac-type, Tianjin Daying Resin Co., Tianjin, China) was mixed in anhydrous ethanol, with mass ratios varying from $2 / 8$ to $8 / 2$, which were calculated on the basis of the mass of $\mathrm{MgO}$, which was expected to be formed from $\mathrm{MgCi}$, against $\mathrm{PF}$ itself.

After drying, the mixture of $\mathrm{MgCi}$ and $\mathrm{PF}$ was heattreated in a horizontal furnace with a heating rate of $5^{\circ} \mathrm{C} /$ min up to $900^{\circ} \mathrm{C}$ in a nitrogen atmosphere and maintained at this temperature for $1 \mathrm{~h}$. The carbonized products were washed by stirring with $500 \mathrm{~mL}$ of $1 \mathrm{~mol} \mathrm{~L}^{-1}$ hydrochloric acid solution at $100^{\circ} \mathrm{C}$ for $2 \mathrm{~h}$ and then leached with distilled water for several times until they were free of chloride ion. The leached products were then dried in vacuum at $120^{\circ} \mathrm{C}$ for $12 \mathrm{~h}$. The products were ground to a size of less than $48 \mu \mathrm{m}$ before being subjected to characterization and electrode preparation. The carbons prepared are called phenolic-resin-based MgO-templated carbons and coded MCPFab, where $\mathrm{ab}$ is the $\mathrm{MgO} / \mathrm{PF}$ mass ratio used. For example, the carbon prepared from the mixture with $\mathrm{MgO} / \mathrm{PF}$ mass ratio of $2 / 8$ is denoted MCPF28.

\subsection{Structural characterization}

Pore characteristics of the resultant carbons were measured with a gas adsorption analyzer (Micromeritics Instrument Corporation Tristar 3000) using $\mathrm{N}_{2}$ at $77 \mathrm{~K}$. BET surface area $S_{\mathrm{BET}}$ was calculated from the isotherm in the relative pressure range $0.05-0.30$. The micropore volume $V_{\text {micro }}$ and the total pore volume $V_{\text {total }}$ were calculated from the amount of $\mathrm{N}_{2}$ adsorbed at a relative pressure $\left(P / P_{0}\right)$ of 0.1 and 0.95 , respectively, using t-plot method. Besides, total pore area $S_{\text {total }}$ and micropore area $S_{\text {micro }}$ were also determined by t-plot method, and mesopore area $S_{\text {meso }}$ was calculated as a balance between $S_{\text {total }}$ and $S_{\text {micro }}$. Pore size distributions were determined by the Barrett Joyner Halenda (BJH) model. Nanostructure of carbons was examined with a transmission electron microscope (TEM, Philips Tecnai G2F-20). Thermogravimetric (TG) analysis was performed on $\mathrm{MgCi}, \mathrm{PF}$ and their mixtures by using a thermogravimetric analyzer (TA Instruments TA-50) under $\mathrm{N}_{2}$ atmosphere.

\subsection{Electrochemical characterization}

EDLC electrodes were prepared by mixing the carbon sample with acetylene black as an electrical conductor and polytetrafluoroethylene (PTFE) as a binder in a mass ratio of 80:10:10, using nickel foam as a current collector. The electrolyte was 30 mass\% $\mathrm{KOH}$. Cyclic voltammetry (CV) measurement was performed on a potentiostat/galvanostat (Princeton Applied Research PARSTAT 2273) with a potential scan rate of $2-50 \mathrm{mV} \mathrm{s}^{-1}$. The capacitance was determined by constructing symmetric coin-type cell with a charge/ discharge battery-measurement system (Land CT2001A, Wuhan, China) with a potential window of $0-1 \mathrm{~V}$. Based on the results of charge-discharge cycling, the specific discharge capacitance of a single electrode in the capacitors can be calculated according to the following equation:

$$
C=(I \cdot \Delta t) /(\Delta V \cdot m),
$$

where $I$ refers to the discharge current, $\Delta t$ to the discharge time, $\Delta V$ to the change of voltage, and $m$ to the mass of resultant carbon on the single electrode. Performance rating of the capacitors was evaluated by the ratio of the capacitance measured at $1000 \mathrm{~mA} \mathrm{~g}^{-1}$ against that at $50 \mathrm{~mA} \mathrm{~g}^{-1}, C_{1000} / C_{50}$. All electrochemical measurements were carried out at room temperature.

\section{Results}

\subsection{Characteristics of the porous carbons used in electrodes}

Figure 1 shows TEM images of a pristine mixture of $\mathrm{MgCi}$ and $\mathrm{PF}$ with $\mathrm{MgO} / \mathrm{PF}$ mass ratio of 8/2 (a), after carbonization (b) and after dissolution of $\mathrm{MgO}$ (c and d). The particles emphasized by a white circle in Figure 1 (a) and (b) correspond to $\mathrm{MgCi}$ and $\mathrm{MgO}$, respectively. Dispersion of the particles seemed to be more uniform in the carbonized sample than in the pristine mixture. Although the crystalline particles of $\mathrm{MgCi}$ has sharp edges (Figure 1(a)), its decomposition product $\mathrm{MgO}$ is changed to be round particles (Figure 1(b)). This process can be illustrated by TG curves shown in Figure 2, along with the probable pyrolysis reaction of $\mathrm{MgCi}$ shown in formula (1) [16]. Three weight loss peaks for $\mathrm{MgCi}$ pyrolysis are evident, between $100-200^{\circ} \mathrm{C}$, $300-400^{\circ} \mathrm{C}$, and $400-600^{\circ} \mathrm{C}$, respectively: the first peak indicates the removal of crystal water, the second one the decomposition of $\mathrm{MgCi}$ to $\mathrm{Mg}$ carbonate, and the third one the formation of $\mathrm{MgO}$. On phenolic resin, its decomposition occur gradual up to around $800^{\circ} \mathrm{C}$ to become carbonaceous solids. The mixture of $\mathrm{MgCi}$ with phenol also shows three weight loss peaks, similar to $\mathrm{MgCi}$, but each peak is shifted to lower temperature side by about $50^{\circ} \mathrm{C}$. These peaks are reasonably supposed to be formed due to the decomposition of $\mathrm{MgCi}$ and the shift happened as a result of the influence of phenolic resin.

$$
\begin{aligned}
& \mathrm{Mg}_{3}\left(\mathrm{C}_{6} \mathrm{H}_{5} \mathrm{O}_{7}\right)_{2} \cdot 14 \mathrm{H}_{2} \mathrm{O} \underset{-\mathrm{I}, 100-200^{\circ} \mathrm{C}}{-16 \mathrm{H}_{2} \mathrm{O}} \mathrm{Mg}_{3}\left(\mathrm{C}_{6} \mathrm{H}_{3} \mathrm{O}_{6}\right)_{2} \\
& \stackrel{\text { II, } 300-400^{\circ} \mathrm{C}}{-3 \mathrm{H}_{2} \mathrm{O},-9 \mathrm{C}} 3 \mathrm{MgCO}_{3} \stackrel{\text { III, } 400-600^{\circ} \mathrm{C}}{-3 \mathrm{CO}_{2}} 3 \mathrm{MgO} \\
& \text { (in } \mathrm{N}_{2} \text { atmosphere) }
\end{aligned}
$$




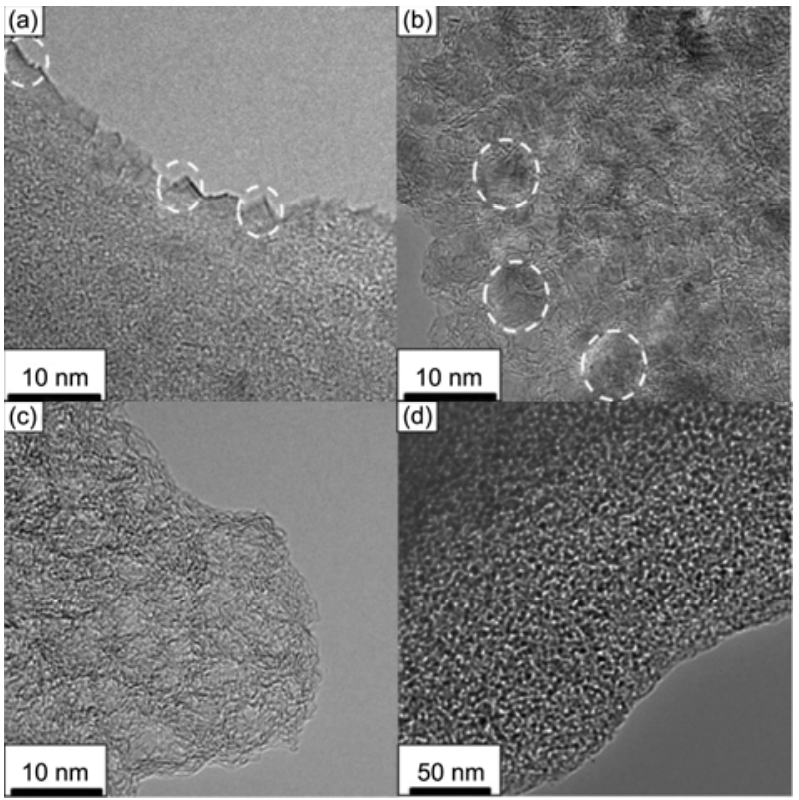

Figure 1 TEM images of the mixture of phenolic resin and magnesium citrate with $\mathrm{MgO} / \mathrm{PF}$ ratio of $8 / 2$. (a) Before carbonization, (b) after carbonization at $900^{\circ} \mathrm{C}$, (c) and (d) after dissolving $\mathrm{MgO}$ (MgO-templated carbon).

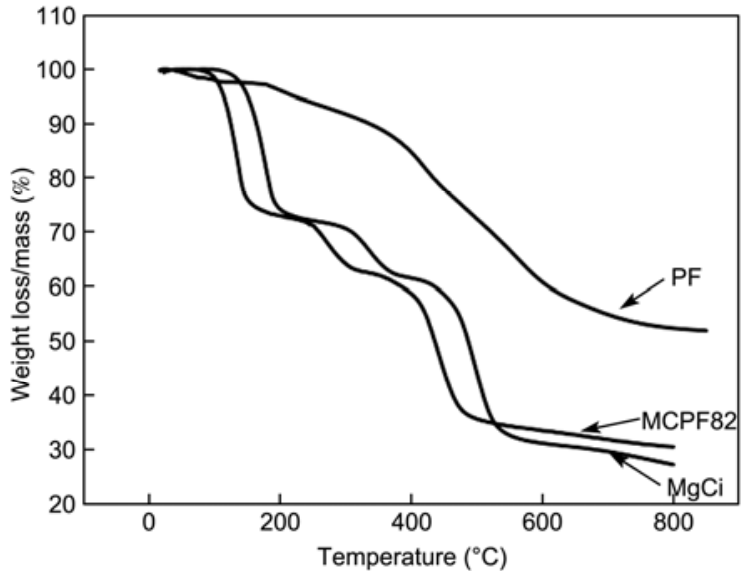

Figure 2 Thermogravimetric curves for $\mathrm{Mg}$ citrate $(\mathrm{MgCi})$, phenolic resin $(\mathrm{PF})$ and their mixture with $\mathrm{MgO} / \mathrm{PF}$ ratio of 8/2 (MCPF82).

Figure 3(a) presents the nitrogen adsorption/desorption isotherms of the resultant carbons measured at $77 \mathrm{~K}$ and the pore parameters determined from these isotherms are listed
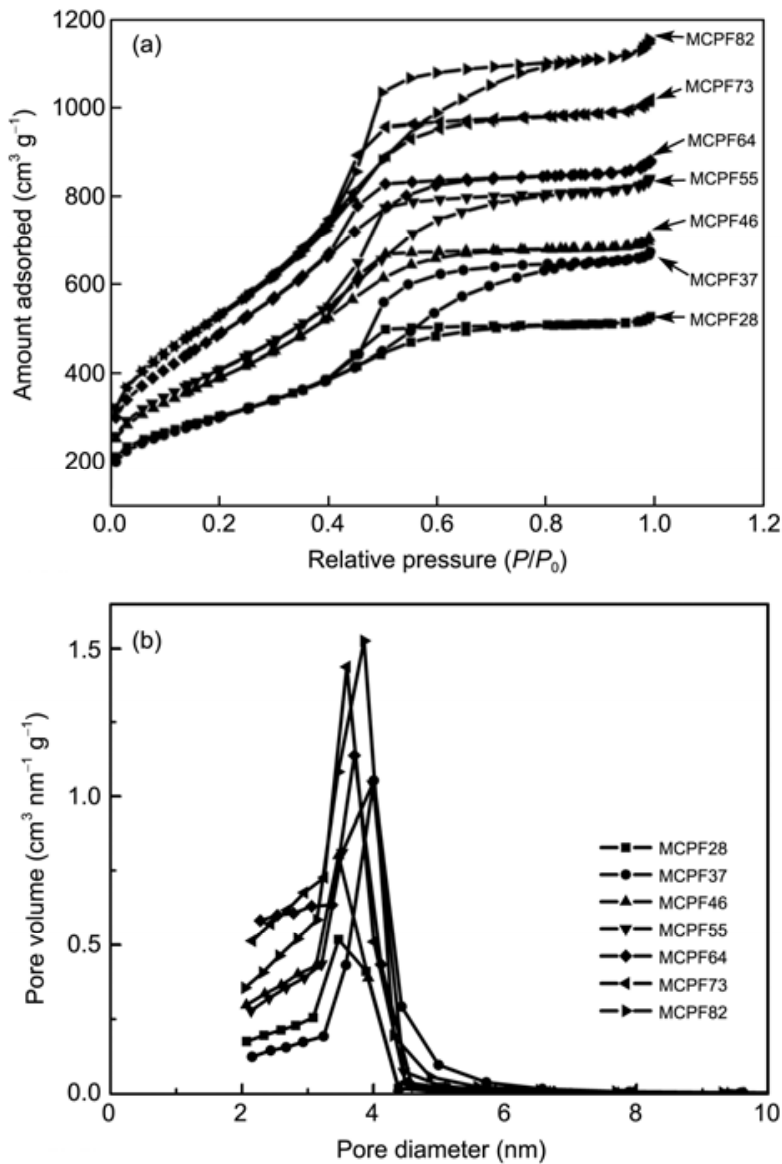

Figure 3 (a) Nitrogen adsorption-desorption isotherms and (b) pore size distribution in the region of mesopores for the $\mathrm{MgO}$-templated carbons.

up in Table 1. As shown in Figure 3(a), all the samples exhibit type-IV isotherms with hysteresis loops caused by capillary condensation in mesoporous structure, revealing that the pores in all the samples are structured mainly by mesopores. Figure 3(b) shows that the size distribution of mesopores is very sharp in these carbons, in other words, the carbons have uniform mesopore size of about $4 \mathrm{~nm}$. In addition, all the isotherms reveal a considerable adsorption at relative low pressure, which is an indication of certain amount of microporosity.

As can be seen from Table 1, all pore parameters determined, $S_{\mathrm{BET},} S_{\text {total }}, S_{\text {meso }}, S_{\text {micro }}, V_{\text {micro }}$, and $V_{\text {total }}$, increase with

Table 1 Pore parameters calculated from $\mathrm{N}_{2}$ adsorption isotherms by the BJH method

\begin{tabular}{cccccccc}
\hline Sample & $S_{\text {BET }}\left(\mathrm{m}^{2} \mathrm{~g}^{-1}\right)$ & $S_{\text {total }}\left(\mathrm{m}^{2} \mathrm{~g}^{-1}\right)$ & $S_{\text {meso }}\left(\mathrm{m}^{2} \mathrm{~g}^{-1}\right)$ & $S_{\text {micro }}\left(\mathrm{m}^{2} \mathrm{~g}^{-1}\right)$ & $S_{\text {meso }} / S_{\text {total }}(\%)$ & $V_{\text {micro }}\left(\mathrm{cm}^{3} \mathrm{~g}^{-1}\right)$ & $V_{\text {total }}\left(\mathrm{cm}^{3} \mathrm{~g}^{-1}\right)$ \\
\hline MCPF28 & 1037 & 1039 & 894 & 145 & 86.04 & 0.117 & 0.829 \\
MCPF37 & 1066 & 1086 & 936 & 150 & 86.19 & 0.149 & 1.219 \\
MCPF46 & 1393 & 1386 & 1228 & 158 & 88.60 & 0.153 & 1.276 \\
MCPF55 & 1460 & 1479 & 1269 & 210 & 85.80 & 0.244 & 1.686 \\
MCPF64 & 1760 & 1752 & 1471 & 281 & 83.96 & 0.286 & 1.863 \\
MCPF73 & 1874 & 1852 & 1552 & 300 & 83.80 & 0.331 & 2.120 \\
MCPF82 & 1920 & 1902 & 1569 & 333 & 82.49 & 0.384 & 2.334 \\
\hline
\end{tabular}


increasing mass ratio of $\mathrm{MgO} / \mathrm{PF}$ and all reach a maximum at the mass ratio of $\mathrm{MgO} / \mathrm{PF}=8 / 2$. As for the ratio of $S_{\text {meso }} /$ $S_{\text {total }}$, it increases at first and then goes down with increasing $\mathrm{MgO} / \mathrm{PF}$ mass ratio, maximizing at the ratio of 4/6. Moreover, from both the point view of pore area and pore volume, the amount of mesopores is far more abundant than micropores.

\subsection{Performance of capacitors}

In Figure 4 (a), voltage sweep CV curves with a sweep rate of $2 \mathrm{mV} \mathrm{s}^{-1}$ are shown for phenolic resin based $\mathrm{MgO}$-templated carbons, revealing that the present carbons show rectangular-shaped (mirror-imaged) $I-V$ curves. No pronounced reversible redox peaks attributed to pseudocapacitance are evident. These results indicate that the charge-discharge responses of the electric double layer are highly reversible in discharge over the same potential range as that required to store the energy on charging. Figure 4(b) presents the CV curves for the sample MCPF82 with various sweep rates changing from 2 to $50 \mathrm{mV} \mathrm{s}^{-1}$. As the scan rate increases, the $\mathrm{CV}$ curves become gradually depressed but still maintain rectangular-shaped characteristics, suggesting that a good accessibility of the electrolyte ions to the carbon surface used in two electrodes and an excellent capacitive behavior of the present carbons even in quick charge-discharge operations.

As expected from rectangular $\mathrm{CV}$ curves, $I-V$ relations on charge-discharge experiment could be approximated to be linear and no potential drop was observed for all carbons tested. In Figure 4(c), the dependence of EDLC capacitance on current density during discharging is shown for the carbons. The decrease in capacitance with increasing current density is rather small for all carbons, and capacitance keeps a constant at current density of more than $250 \mathrm{~mA} \mathrm{~g}^{-1}$.

In Table 2, EDLC capacitances (current density $=50$ or $1000 \mathrm{~mA} \mathrm{~g}^{-1}$ ) together with the performance rating defined by $C_{1000} / C_{50}$ are listed for the resultant carbons. With growing $S_{\mathrm{BET}}$, both $C_{50}$ and $C_{1000}$ increase and reach a maximum at $\mathrm{MgO} / \mathrm{PF}=8 / 2$, fitting with the general understanding that high surface area is preferable to high capacitance. Capacitance retentions are all above $92 \%$. Moreover, comparing two sets of data, $S_{\text {meso }} / S_{\text {total }}$ in Table 1 and $C_{1000} / C_{50}$ in Table 2 , the performance rating $C_{1000} / C_{50}$ follows the same trend with the $S_{\text {meso }} / S_{\text {total }}$ ratio, maximizing at mass ratio of $\mathrm{MgO} /$ $\mathrm{PF}=4 / 6$ with the value of $98.3 \%$, although $\mathrm{S}_{\mathrm{BET}}$ of the sample MCPF46 was merely $1393 \mathrm{~m}^{2} \mathrm{~g}^{-1}$.

\section{Discussion}

\subsection{Formation of mesoporous carbons}

It is known that the surface of $\mathrm{MgCi}$ particles is covered by plasticized resin during dehydration process (step I in formula (1)). The coating of $\mathrm{MgCi}$ particles by resinous materials is reasonably supposed to inhibit the grain growth of $\mathrm{MgCO}_{3}$
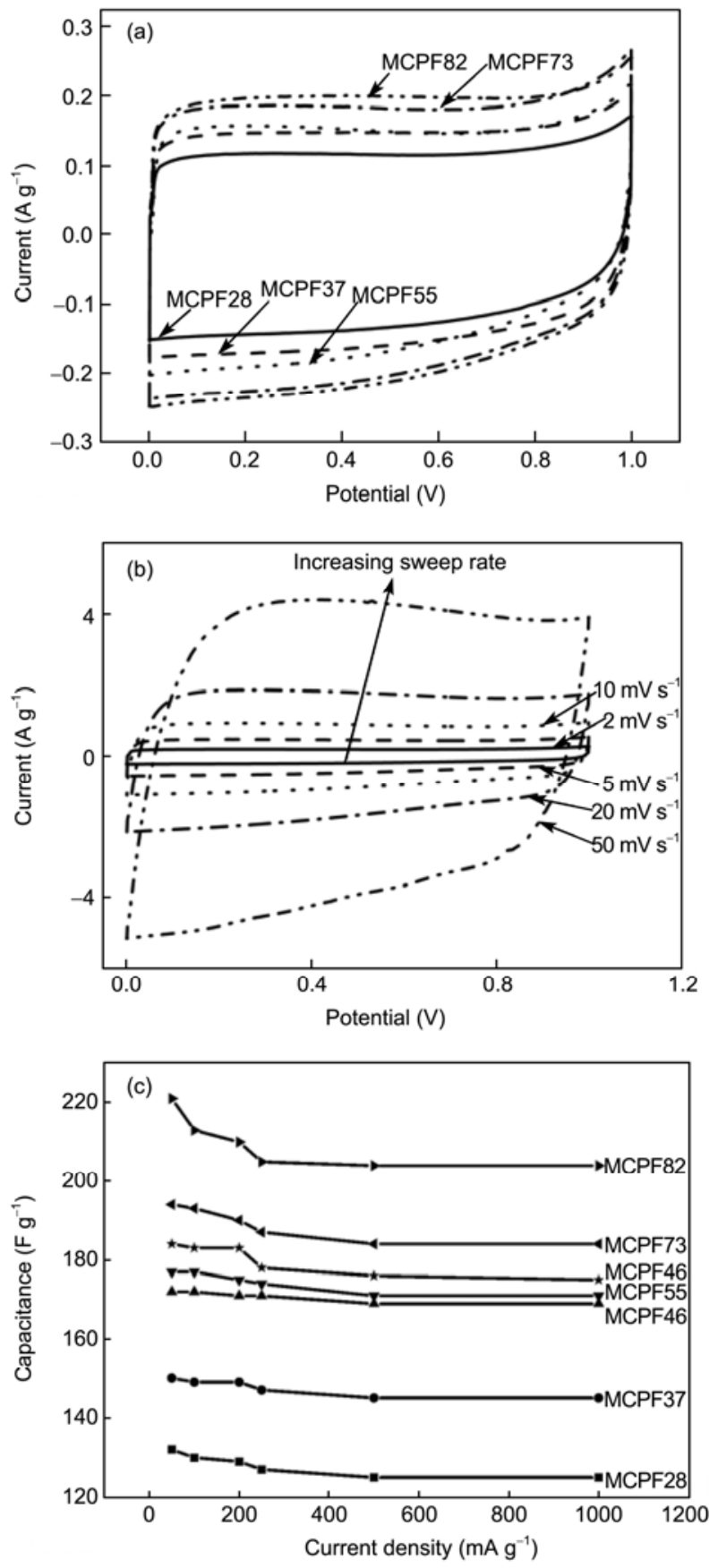

Figure 4 (a) $\mathrm{CV}$ curves obtained from the $\mathrm{MgO}$-templated carbons at a scan rate of $2 \mathrm{mV} \mathrm{s}^{-1}$, (b) those on the sample MCPF82 at different scan rates, and (c) the rate performance of $\mathrm{MgO}$-templated carbons.

and $\mathrm{MgO}$ particles during further decomposition (step II and III in formula (1)). In addition, $\mathrm{MgCO}_{3}$ yielded from $\mathrm{MgCi}$ prevents further agglomeration of $\mathrm{MgO}$ particles. This was the reason why the decomposition product $\mathrm{MgO}$ could keep geometric identity, uniform size of ca. $4 \mathrm{~nm}$ and uniform morphology, as shown in Figure 1 (b). A comparison of Figure 1 (a) and (b) shows that the edges of crystal decrease from (a) to (b), which reveals that a crystal transfer happened during the decomposition process of $\mathrm{MgCi}$. This 
Table 2 EDLC capacitances at different charging rates and rate performance for the carbons obtained

\begin{tabular}{cccc}
\hline Sample & $C_{50}\left(\mathrm{~F} \mathrm{~g}^{-1}\right)$ & $C_{1000}\left(\mathrm{~F} \mathrm{~g}^{-1}\right)$ & $C_{1000} / C_{50}(\%)$ \\
\hline MCPF28 & 133 & 125 & 94.1 \\
MCPF37 & 150 & 145 & 96.7 \\
MCPF46 & 172 & 169 & 98.3 \\
MCPF55 & 177 & 171 & 96.6 \\
MCPF64 & 184 & 175 & 95.1 \\
MCPF73 & 194 & 184 & 94.8 \\
MCPF82 & 221 & 204 & 92.2 \\
\hline
\end{tabular}

result is in accordance with formula (1), which exhibits the decomposition process of $\mathrm{MgCi}$ into $\mathrm{MgO}$. Removal of the $\mathrm{MgO}$ particles left mesopores of homogeneous size and morphology afterwards, as shown by TEM image in Figure 1(c) and by pore size distribution in Figure 3(b).

Since mesopores are formed as the heritages of $\mathrm{MgO}$ nanoparticles, the amount of mesopores grows with the increasing amount of $\mathrm{MgCi}$ mixed. However, it has to be pointed out that some amount of micropores is formed, particularly in the carbon prepared from the mixtures with high $\mathrm{MgO} / \mathrm{PF}$ ratios (Table 1). Micropores, which existed principally on the wall of mesopores, however, are supposed to have two resources, citric acid in $\mathrm{MgCi}$ and the starting resin PF. As illustrated by the TG curves in Figure 2, the generation of $\mathrm{MgO}$ occurs at about $470^{\circ} \mathrm{C}$ followed by the final generation of carbonaceous residues from $\mathrm{PF}$, resulting in the complete coating of $\mathrm{MgO}$ nanoparticles by carbonaceous residues formed from citric acid and PF. Therefore, the shrinkage of carbon is supposed to be hindered in the carbonaceous film coated the $\mathrm{MgO}$ nanoparticles up to $800^{\circ} \mathrm{C}$ and the movement of carbonaceous residues during their carbonization above $800^{\circ} \mathrm{C}$ is strongly limited. Accompanying the formation of residues, evolvement and liberation of gases like $\mathrm{CO}_{2}, \mathrm{H}_{2} \mathrm{O}$ from both $\mathrm{MgCi}$ and $\mathrm{PF}$ occurred, and thus micropores were created. This is supposed to follow the mechanism of physical activation by these gases.

Because all adsorption/desorption isotherms of $\mathrm{N}_{2}$ show marked hysteresis loops, they belong to physisorption isotherms type IV and the hysteresis loops belong to type-H2 according to IUPAC [17] and related to the capillary condensation associated with ink-bottle-type pores, the simplest image of which is a spheroidal cavity fitted with one or several narrow cylindrical necks. Pore structure model deducted from hysteresis loops is also found to be in accordance with pore formation process: micropores, which existed in the carbonaceous residues formed from two precursors, $\mathrm{MgCi}$ and phenolic resin, are supposed to be connected to the $\mathrm{MgO}$ crystal after the completion of whole decomposition process of two precursors, and consequently they were directly connected to mesopores after the removal of $\mathrm{MgO}$, forming a pore network structure, just like necks of ink bottle connect to the cavity.

\subsection{Capacitor performance of mesoporous carbons}

With the mesoporous carbons prepared using as electrode material, electric double-layers were formed even with a sweep rate of $50 \mathrm{mV} \mathrm{s}^{-1}$ and no evidence for pseudocapacitance is observed on CV curves (Figure 4(b)). Moreover, good rate performance of more than $92.2 \%$ by using performance rating $C_{1000} / C_{50}$ is got. This result might be due to the short ion transport paths through the ink-bottle mesopores to the micropores.

The capacitance of the capacitors, however, does not have a linear relationship with their specific surface area

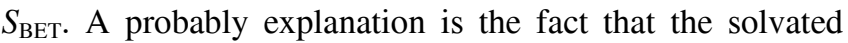
electrolyte ions are too big to adsorb into some micropores, which lead to low utilization rate of surface area. Thus, the surface area of these non-accessible micropores will not contribute to the total EDLC capacitance of the electrode materials [18,19]. The carbon MCPF46, which got the highest ratio of $S_{\text {meso }} / S_{\text {total }}$, shows the highest retention in capacitance, performance rating $C_{1000} / C_{50}$ reaching $98.3 \%$, even though the capacitance is not the highest. This may suggest a successful match of mesopores and micropores, namely a best balance between double-layer contributor and ion-channel contributor, the former increasing capacitance, and the latter enhancing rate performance of capacitors.

Considering that $\mathrm{MgCi}$ and $\mathrm{PF}$ are both cheap industrial product, the preparation of carbons was carried out without any stabilization and activation process, and the template $\mathrm{MgO}$ was experimentally demonstrated to be recycled [10]. Thus this method is a promising way to prepare electrode material of EDLC for its price advantage.

\section{Conclusions}

Templated mesoporous carbons with surface area around $1400 \mathrm{~m}^{2} \mathrm{~g}^{-1}$ were prepared from mixtures of thermoplastic phenolic resin and $\mathrm{MgCi}$ only through carbonization. $\mathrm{MgO}$, the pyrolysis product of $\mathrm{MgCi}$, dispersed uniformly in the carbonized samples and ensured the uniform size of mesopores. According to the pore formation process and $\mathrm{N}_{2}$ adsorption isotherms, pore morphology is supposed to be inkbottle, whose cavities correspond to mesopores, and the necks to micropores. The performance of the samples in electrochemical capacitors is essentially based on electric double-layer mechanism. The pore structure facilitates the ion transport and enhances the rate performance of capacitors. The values of performance rating reach a maximum of $98.3 \%$, expressed in the form of $C_{1000} / C_{50}$. As far as the correlation between the cost and the specific capacitance is concerned, the carbons obtained by the carbonization of mixtures of phenolic resin with magnesium citrate seem to be promising material for electrode of EDLC. 
This work was supported by the Natural Science Foundation of Tianjin City (12JCZDJC27000) and the National Natural Science Foundation of China (51172160). The authors would like to express their thanks especially to the support and comments of Prof. Toyoda Masahiro of Oita University, Japan.

1 Frackowiak E, Béguin F. Carbon materials for the electrochemical storage of energy in capacitors. Carbon, 2001, 6: 937-950

2 Inagaki M, Konno H, Tanaike O. Carbon materials for electrochemical capacitors. J Power Sources, 2010, 24: 7880-7903

3 Lee J, Kim J, Hyeon T. Recent progress in the synthesis of porous carbon materials. Adv Mater, 2006, 16: 2073-2094

4 Lee J S, Joo S H, Ryoo R. Synthesis of mesoporous silicas of controlled pore wall thickness and their replication to ordered nanoporous carbons with various pore diameters. J Am Chem Soc, 2002, 7: 1156-1157

5 Joo S H, Choi S J, Oh I, et al. Ordered nanoporous arrays of carbon supporting high dispersions of platinum nanoparticles. Nature, 2001, 6843: $169-172$

6 Inagaki M, Miura $\mathrm{H}$, Konno $\mathrm{H}$. A new simple process for carbon coating of ceramic particles using poly (vinyl chloride). J Eur Ceram Soc, 1998, 8: 1011-1015

7 Konno H, Oyamada K, Inagaki M. Formation of carbonaceous-zcoatings on aluminium using poly (vinyl alcohol). J Eur Ceram Soc, 2000, 9: 1391-1396

8 Inagaki M, Okada Y, Miura H, et al. Preparation of carbon-coated transition metal particles from mixtures of metal oxide and polyvinylchloride. Carbon, 1999, 2: 329-334

9 Morishita T, Soneda Y, Tsumura T, et al. Preparation of porous carbons from thermoplastic precursors and their performance for electric double layer capacitors. Carbon, 2006, 12: 2360-2367

10 Morishita T, Tsumura T, Toyoda M, et al. A review of the control of pore structure in $\mathrm{MgO}$-templated nanoporous carbons. Carbon, 2010, 10: $2690-2707$

11 Inagaki M, Kobayashi S, Kojin F, et al. Pore structure of carbons coated on ceramic particles. Carbon, 2004, 15: 3153-3158

12 Morishita T, Ishihara K, Kato M, et al. Preparation of a carbon with a $2 \mathrm{~nm}$ pore size and of a carbon with a bi-modal pore size distribution. Carbon, 2007, 1: 209-211

13 Inagaki M, Kato M, Morishita T, et al. Direct preparation of mesoporous carbon from a coal tar pitch. Carbon, 2007, 5: 1121-1124

14 Konno H, Onishi H, Yoshizawa N, et al. MgO-templated nitrogencontaining carbons derived from different organic compounds for capacitor electrodes. J Power Sources, 2010, 2: 667-673

15 Wang Y S, Wang C Y, Chen M M. New mesoporous carbons prepared from pitch by simultaneous templating and carbonization. New Carbon Mater, 2009, 2: 187-190

16 Mansour S A A. Thermal decomposition of magnesium citrate 14-hydrate. Thermochimica Acta, 1994, 2: 231-242

17 Sing K S W. Reporting physisorption data for gas/solid systems with special reference to the determination of surface area and porosity. Pure Appl Chem, 1985, 4: 603-619

18 Arulepp M, Permann L, Leis J, et al. Influence of the solvent properties on the characteristics of a double layer capacitor. J Power Sources, 2004, 2: 320-328

19 Huang J, Sumpter B G, Meunier V. A universal model for nanoporous carbon supercapacitors applicable to diverse pore regimes, carbon materials and electrolytes. Chem Eur J, 2008, 22: 6614-6626

Open Access This article is distributed under the terms of the Creative Commons Attribution License which permits any use, distribution, and reproduction in any medium, provided the original author(s) and source are credited. 